\title{
Measurement of subjective and objective indices after progressive resistance training compared with aerobic training in Patients with
}

\section{Haemophilia: a study protocol}

Running title: effects of exercise in hemophilia

Name:

Designation:

Department:

Sciences

Qualification:

Email address:

Name:

Designation:

Department:

Qualification:

Email address:

Name:

Designation:

Department:

Qualification:

Email address:
Felicianus Anthony Pereira (Corresponding author)

Senior Lecturer

National Institute of Physical Therapy \& Rehabilitation

Doctor of Physical Therapy

f.pereira93@hotmail.com

Dr. Nabila Najam Soomro

Professor

Sindh Institute of Physical Medicine and Rehabilitation

FCPS

nabila61@gmail.com

Farheen Sulaiman

Program Coordinator, MSAPT, Assistant Professor

Sindh Institute of Physical Medicine and Rehabilitation

MSc

farheen.sulaiman@fulbrightmail.org 


\section{ABSTRACT}

Background: Bleeding episodes in mild haemophilia may occur after major injuries or surgical procedures with some people not experiencing bleeding episodes. People suffering from moderate haemophilia bleed once a month, however they rarely experience spontaneous bleeding. Those suffering from severe haemophilia bleed quite often into muscles or joints, and episodes can occur once to twice a week. Bleeding usually occurs spontaneously.

Objective: To investigate the effects of progressive resistance training on quality of life, muscular strength and joint score in patients with haemophilia.

Methodology: Sixty patients will be enrolled in the study. Thirty patients will be allocated to control group, and thirty to intervention group. Controls will be administered active muscle stretching and aerobic exercises. Intervention group will be given active muscle stretching, and resistance training. Patients will be randomly allocated to each group. Anthropometric data will be measured pre-test to establish a baseline. Study variables include muscular strength, and quality of life. All tests will be measured pre-test and post-test to compare effects of treatment.

Results: Participant recruitment commenced in June 2021. The post intervention phase will be completed by August 2020. Data analysis will commence after this. A write-up for publication is expected to be completed after the follow-up phase is finalized in August 2021.

Conclusions: If resistance training is found to be effective in improving quality of life and muscular strength in participants, it could reduce the frequency of factor therapy given prophylactically, or even as acute treatment, thus directing it towards more severe cases. It will also provide financial relief to organizations supporting the treatment of the hemophilic population.

Keywords: Hemophilia, resistance training, aerobic exercise, Pakistan 
medRxiv preprint doi: https://doi.org/10.1101/2022.01.27.22269981; this version posted January 30, 2022. The copyright holder for this preprint

\section{1. INTRODUCTION}

2 Haemophilia is an X-linked congenital bleeding disease which occurs due to lack coagulation

3 factors VIII or factor IX. This deficit is an effect of mutations in clotting factor genes (FVIII

4 in type A, and FIX in type B). Occurrence of haemophilia is approximately 1 in 10000

5 births. ${ }^{1}$ Haemophilia A is found more commonly in the population. Regardless of the type of

6 haemophilia, the outcome is the same; people affected bleed longer than normal. In the

7 healthy population, normal international units (IU) are $0.50-1.50$ per milliliter of whole

8 blood. People with mild haemophilia have levels of 0.05-0.4 IU, and people with moderate

9 haemophilia have levels of 0.01-0.05 IU. Severe is below $0.01 \mathrm{IU}^{2}$ People with mild

10 haemophilia might experience bleeding after surgical procedures or major injury, while

11 others may not experience bleeding episodes. People with moderate haemophilia bleed

12 around once a month and rarely experience spontaneous bleeding. Those suffering from

13 severe haemophilia bleed quite often into muscles or joints, and episodes might occur once or

14 twice a week. Bleeding usually occurs spontaneously. ${ }^{2}$ In the annual global survey

15 conducted across 116 countries by World Federation of Hemophilia (WFH) in 2017, a total

16 of 196,706 people have been identified with haemophilia. In Pakistan, 1743 people were

17 diagnosed with haemophilia, 250 had Von Willebrand's disease, and 121 had other associated

18 bleeding disorders. ${ }^{3}$ Psychosocial factors play a key role in quality of life (QoL) of patients

19 with haemophilia $(\mathrm{PwH}){ }^{4,5}$ As severity of hemophilia increases, the QoL in PwH decreases

20 when compared with healthy population. ${ }^{6}$ Self-esteem of males who have severe bleeding

21 disorders is also considerably worse compared to that of their healthy peers, whereas females

22 show significant differences. ${ }^{7}$ Treatment for haemophilia is via factor replacement therapy,

23 which may be given prophylactically, or as acute treatment. ${ }^{8}$ Hemophilic arthropathy can be

24 extremely painful, with knees, elbows and ankle most commonly being affected. Recurring

25 bleeding in the joint can cause synovitis that can develop into arthropathy, which may later 
26 disrupt activities of daily living. The joint most commonly affected is the knee, with a simple

27 act such as ambulating on level surfaces generating substantial force and stresses on the knee

28 joint. Due to muscle weakness, the knee joint is further susceptible to weight bearing stresses,

29 which results in a loop of persistent joint bleeds and rising synovitis which causes further

30 muscle atrophy, eventually leading to severe arthropathy. ${ }^{9}$ Traditionally PwH were often

31 advised against participating in sports due to possibility of injury, however, studies have tried

32 to demonstrate the positive effects of exercise programs for $\mathrm{PwH}$.

\section{Methods}

34 The study design is a single blind, randomized controlled trial, and study setting is single

35 center study. Ethical approval has been obtained from institutional review board (Ref no.

36 IRB-1936/DUHS/Approval/2021/), and has been registered as a clinical trial in

37 clinicaltrials.gov on $19^{\text {th }}$ May, 2021 (identifier: NCT04892628). Sample size was calculated

38 using OpenEpi version 3.0 calculator. In the section of overall HJHS score of resistance

39 training group ${ }^{(14)}$, the detectable difference and clinically important difference ranged from

$407.25 \pm 1.28$ and $4.83 \pm 1.19$ points. A power of $80 \%$ and confidence interval of $95 \%$ was set and

41 a sample size of 5 per group was obtained. However, the sample size will be increased to 30

42 participants per group.

\section{Inclusion Criteria}

44 The study will include participants:

- clinically diagnosed with hemophilia A or B (mild to moderate hemophilia (mild

46 hemophilia - 0.05-0.4 IU, moderate hemophilia - 0.01-0.05 IU)

- willing to undergo pre- and post-program objective assessment of manual muscle

- willing to train two times a week 
- males and females between 18 to 45 years of age.

- those who can ambulate without assistance

\section{Exclusion Criteria}

- unable to attend exercise sessions for the complete duration of the study

- $\quad$ those who have had surgery performed in the past six weeks, or are scheduled for surgery in duration of training

- involvement in any other training, or rehabilitation, during study

- $\quad$ change in medicine within the study

- $\quad$ history of major bleeding episodes that could pose a risk

- History of FVIII or FIX inhibitor

\section{Data collection}

62 Data will be collected from a Hemophilia Welfare center located in Karachi, Pakistan. A

63 hematologist, orthopedic and general physician, as well as paramedical staff are present on-

64 site, to guarantee safety of patients. Sixty participants will be included in the study. Written

65 informed consent will be obtained from participants, with forms being provided in both

66 English and in Urdu. Once consent has been obtained, participants will be enrolled in the

67 study. Participants will be divided equally in two groups: a control group and an intervention

68 group. Allocation to both these groups will be done by simple random sampling, using a

69 computer software (randomizer.org), with participants unaware regarding group allocation.

70 Participant data is available via the registry maintained by the welfare center, providing a

71 sample frame from which participants can be selected. 
75 Participant's height, weight and age will be recorded for pre-test anthropometric data. Height

76 will be measured using a stadiometer, and weight will be measured with an electronic scale.

77 Hemophilia Joint Health Score v2.1 and Manual Muscle Testing (Daniels and

78 Worthingham's) for deltoid (anterior, middle and posterior fibers), biceps, triceps,

79 quadriceps, hamstrings, and calf muscles will be conducted pre and post-test. HEP-Test-Q

80 will be assessed pre and post-test to note change in subjective QoL. Rating of perceived

81 exertion (Borg rating of perceived exertion) will be assessed on every session to check

82 progression in variables being tested. HEP-Test-Q and Rating of perceived exertion will be

83 provided in Urdu for participants. Treatment sessions will be conducted twice a week, for

84 eight weeks, amounting to a total of sixteen sessions. The control group will be administered

85 a standard physical therapy intervention program which will consist of active muscle

86 stretching and aerobic exercise. License has been obtained from the relevant authorities to use

87 these questionnaires in this study.

88 Exercises will include:

- Flexion, extension and abduction at the shoulder joint for anterior, middle and posterior fibers of deltoid respectively

- Flexion and extension at the elbow for biceps brachii and triceps trachii

- Flexion and extension at the knee joint for quadriceps and hamstring muscles

- Plantar flexion at the ankle joint for gastrocnemius and soleus

94 Three sets of ten repetitions of each exercise will be done.

95 The intervention group will undergo a progressive resistance training program consisting of

96 active muscle stretching and resistance training. Exercise for intervention group will consist

97 of: 
Price of one injection of FVIII in Pakistan is around USD 80/- ${ }^{10}$. A patient who requires $12-$

12116 injections every month, the cost will be roughly USD 11,500 USD 15,500/- anually. ${ }^{10}$ In

- Resisted flexion, extension and abduction at the shoulder joint for anterior, middle and posterior deltoid, using dumbbells

- Resisted flexion and extension at the elbow for biceps brachii and triceps trachii, using

- Resisted flexion and extension of knee joint for quadriceps and hamstring muscles, using therabands

- Resisted dorsiflexion and plantar flexion of gastrocnemius and soleus, using therabands

Resistance will be increased on basis of progressive overload principle. Three sets of ten repetitions will be performed per muscle group. Initial weight of the dumbbells will be $1 \mathrm{~kg}$ and will be increased by $0.5 \mathrm{~kg}$ per week. Therabands used will be Yellow, Red, Green and Blue. Resistance for therabands will be upgraded every two weeks. Treatment time per group will be approximately forty minutes. Exercises will be progressed in a gradual manner to give participants time to build muscle strength, and prevent chances of injury.

$\underline{\text { Statistical Treatment }}$

\section{Study variables include:}

- Progressive resistance training, gender and age, weight are the independent variable

- Quality of life, muscle strength, rating of perceived exertion and joint score are the dependant variables

\section{Statistical Analysis}

The software for data analysis will be IBM SPSS version 21. Mean and standard deviation will be calculated for qualitative variables. For quantitative variables, paired sample T-test will be applied. 
122 a country where the yearly minimum wage is $\$ 2,484.00 \mathrm{USD}$, affording such treatment

123 becomes difficult for the population.

124 Limitations

125 - Isokinetic dynamometer cannot be used to assess muscular strength due to cost of 126 equipment.

127 Questionnaires such as Haem-A-QoL that required purchasing fees cannot be used

128 - Study setting is a single center study

\section{REVIEW OF LITERATURE}

130 A systematic review was conducted in 2015 by G. S. Schäfer et al in nine controlled clinical

131 trials on pain and musculoskeletal function in PWH. The study found that exercise and

132 physical therapy maneuvers decrease pain perception and increase range of motion and

133 muscular strength in the hemophiliac population. It also noted that further RCTs, with greater

134 emphasis on methodology and greater focus on parameters of exercise prescription are

135 required. ${ }^{11}$ A systematic review on methods and results of psychosocial features of

136 hemophilia displayed that studies into psychosocial aspects of the disease is still lacking.

137 Most of the studies conducted are questionnaire based. Results across studies could not be

138 assessed due to the variety of questionnaires used. PwH are affected by factors which may

139 consist of life satisfaction, self-esteem, anxiety and depression. It was also noted that

140 stressors could differ all through hemophilia life cycle. ${ }^{4}$ A systematic review on benefits of

141 exercise for $\mathrm{PwH}$ found that correctly performed exercise along with involvement in suitable

142 sports are helpful for PwH. Competing in sports provides many physical benefits, along with

143 boosting emotional and societal well-being. The article also points out that participation

144 should be in appropriate sports, as each case of hemophilia is unique. ${ }^{12}$

145 A clinical trial conducted in 2003, of physical training in subjects with hemophilia confirmed

146 specific sports therapy consisting of proprioceptive utility with mild strength exercise 
147 increased the proprioceptive functioning along with muscular power. ${ }^{13}$ A randomized

148 controlled trial conducted in 2016 by Runkel et al on programmed sports therapy, among 64

149 PwH found significant differences in values of triceps, biceps, latissimus dorsi, and

150 quadriceps femoris between control and intervention groups. Outcome measures used in the

151 study included strength measurements, joint score, co-ordination test and 12 minute walk

152 test. ${ }^{14}$ In a study by S El-Shamy on the outcome of whole body vibration in children suffering

153 from hemophilia found significant differences between intervention group and control group.

154 (Quadriceps peak torque $\mathrm{p}<0.001$, functional capacity $\mathrm{p}=0.006) .{ }^{15}$ Data obtained from a study

155 of 32 patients by Greene et al showed that significant increase in muscular strength of flexor

$156(\mathrm{p}<0.0001)$ and extensor $(\mathrm{p}<0.001)$ aspect of the knee can be achieved by an isokinetic

157 training program. Greater improvement was noticed in patients whose severity of arthropathy

158 was lower, who displayed an increase of $1 \mathrm{~cm}$ thigh girth, and who exercised more

159 frequently. ${ }^{9}$

160 A RCT was conducted in 2017 on 20 patients to assess effectiveness of home exercise

161 program in adult PwH. Significant difference was noted between QoL, illness behaviors, and

162 pain perception of ankle; however no significant difference in joint status was noted. The

163 authors concluded that perception of pain can be altered by administering a home exercise

164 plan. ${ }^{16}$ A review article by Stephensen et al on orthopedic co-morbidities in elderly

165 hemophilia population highlighted joint status, pain, muscle atrophy and strength, balance

166 and gait, physical activity and QoL and bone mineral density. The study found that exercise

167 can help slow down normal degeneration processes that occur with ageing. Recommended

168 exercise frequency is two to three times a week, for at least 12 weeks in the ageing

169 hemophilia population. ${ }^{17}$ A cross-sectional study was conducted among $12 \mathrm{PwH}$ (11 severe,

170 and 1 mild) to assess if upper body muscle strength would vary depending on the

171 intervention. Results of the study showed that external resistance of muscles at moderate 
172 intensities, compared to conventional non-resisted therapeutic exercises, provide greater

173 muscle activity than non-resisted exercises. ${ }^{18}$ An observational study on QoL in children with

174 hemophilia and sporting activities was conducted by R. Cuesta-Barriuso et al. fifty three

175 children with hemophilia and fifty one children without were assessed. Exercises were done

176 bi-weekly over 15-weeks. Home exercises were done daily, 6 days a week, Outcome measure

177 used to assess objective measures was the Spanish version of HJHS. No considerable

178 discrepancy was found in perception of QoL among groups. Participation in sports increased

179 the joint health score and QoL in children with hemophilia. ${ }^{19}$ A nationwide population-based

180 study was conducted in Taiwan. Data was obtained from patients who were recorded in the

181 national database. The study set out to assess factors influencing availing of rehabilitation.

182 Total rehabilitation cost stood at $<0.1 \%$ of total yearly medical expenses. Physical therapy

183 accounted for $71.2 \%$ of rehabilitation therapy. The study concluded that surgical procedures

184 related to nervous system and musculoskeletal systems, and higher usage of FVIII affected

185 rehabilitation usage by $\mathrm{PwH}$ A (odds ratio 3.788; $\mathrm{p}<0.001$ ). ${ }^{20}$ A randomized controlled trial

186 conducted in 2012 on therapeutic exercises versus hydrotherapy on pain intensity in PwH

187 both methods decreased pain, and increased range of motion. Hydrotherapy was more

188 effective than exercise in management of pain. Significant difference was not found in range

189 of motion after intervention with these methods. ${ }^{21}$

\section{REFERENCES}

${ }^{1}$ Srivastava A, Brewer AK, Mauser $\square$ Bunschoten EP, Key NS, Kitchen S, Llinas A et al. Guidelines for the management of hemophilia. Haemophilia. 2013;19(1):e1-47.

${ }^{2}$ World federation of hemophilia. Severity of hemophilia. https://www.wfh.org/en/page.aspx ?pid=643 (accessed 10 February 2019).

${ }^{3}$ World Federation of Hemophilia. Annual Global Survey 2017, World Federation of Hemophilia. undefined: 31-4.

${ }^{4}$ Cassis FY, Querol F, Forsyth A, Iorio A, HERO International Advisory Board. Psychosocial aspects of haemophilia: a systematic review of methodologies and findings. Haemophilia. 2012;18(3):e10114.

${ }^{5}$ Witkop M, Guelcher C, Forsyth A, Hawk S, Curtis R, Kelley L et al. Treatment outcomes, quality of life, and impact of hemophilia on young adults (aged 18-30 years) with hemophilia. American journal of hematology. 2015;90:S3-10. 
${ }^{6}$ Barlow JH, Stapley J, Ellard DR. Living with haemophilia and von Willebrand's: a descriptive qualitative study. Patient education and counseling. 2007;68(3):235-42.

${ }^{7}$ Limperg PF, Haverman L, Maurice-Stam H, Coppens M, Valk C, Kruip MJ et al. Health-related quality of life, developmental milestones, and self-esteem in young adults with bleeding disorders. Quality of Life Research. 2018;27(1):159-71.

${ }^{8}$ Chen SL. Economic costs of hemophilia and the impact of prophylactic treatment on patient management. Am J Manag Care. 2016 Apr 1;22(5 Suppl):s126-33.

${ }^{9}$ Greene WB, Strickler EM. A modified isokinetic strengthening program for patients with severe hemophilia. Developmental Medicine \& Child Neurology. 1983;25(2):189-96.

${ }^{10}$ Fatimid Foundation (2013) Cost of treatment, Available at: https://www.fatimid.org/index.php?option=com content $\&$ view=article $\& i d=13 \& I t e m i d=39$ (Accessed: February: 2019).

${ }^{11}$ Schäfer GS, Valderramas S, Gomes AR, Budib MB, Wolff ÁL, Ramos AA. Physical exercise, pain and musculoskeletal function in patients with haemophilia: a systematic review. Haemophilia. 2016;22(3):e119-29.

${ }^{12}$ Negrier C, Seuser A, Forsyth A, Lobet S, Llinas A, Rosas M, Heijnen L. The benefits of exercise for patients with haemophilia and recommendations for safe and effective physical activity. Haemophilia. 2013;19(4):487-98.

${ }^{13}$ Hilberg T, Herbsleb M, Puta C, Gabriel HH, Schramm W. Physical training increases isometric muscular strength and proprioceptive performance in haemophilic subjects. Haemophilia. 2003;9(1):86-93

${ }^{14}$ Parhampour B, Dadgoo M, Vasaghi-Gharamaleki B, Torkaman G, Ravanbod R, Mirzaii-Dizgah I, Baghaipour MR, Saneii SH. The effects of six-week resistance, aerobic and combined exercises on the pro-inflammatory and anti-inflammatory markers in overweight patients with moderate haemophilia A: A randomized controlled trial. Haemophilia. 2019 Jul 1;25(4):e257-66.

${ }^{15}$ El-Shamy S. Effect of whole body vibration training on quadriceps strength, bone mineral density, and functional capacity in children with hemophilia: a randomized clinical trial. Journal of musculoskeletal \& neuronal interactions. 2017;17(2):19.

${ }^{16}$ Cuesta-Barriuso R, Torres-Ortuño A, Nieto-Munuera J, López-Pina JA. Effectiveness of an educational physiotherapy and therapeutic exercise program in adult patients with hemophilia: a randomized controlled trial. Archives of physical medicine and rehabilitation. 2017;98(5):841-8.

${ }^{17}$ Stephensen D, Bladen M, McLaughlin P. Recent advances in musculoskeletal physiotherapy for haemophilia. Therapeutic advances in hematology. 2018;9(8):227-37.

${ }^{18}$ Calatayud J, Pérez-Alenda S, Carrasco JJ, Escriche A, Cruz-Montecinos C, Andersen LL et al. Upper-Body Exercises With External Resistance Are Well Tolerated and Enhance Muscle Activity in People With Hemophilia. Physical therapy. 201923.

${ }^{19}$ Cuesta-Barriuso R, Torres-Ortuño A, Pérez-Alenda S, Carrasco JJ, Querol F, Nieto-Munuera J. Sporting activities and quality of life in children with hemophilia: an observational study. Pediatric physical therapy. 2016;28(4):453-9.

${ }^{20}$ Chen CM, Yang YH, Chang CH, Chen CC, Chen PC. The utilization of rehabilitation in patients with hemophilia A in Taiwan: a nationwide population-based study. PloS one. 2016;11(9):e0164009.

${ }^{21}$ Mazloum V, Rahnama N, Khayambashi K. Effects of therapeutic exercise and hydrotherapy on pain severity and knew range of motion in patients with haemophilia: a randomized controlled trial. International journal of preventive medicine. 2014;5(1):83 